encountered more frequently in the cardiac catheterisation laboratory. Survival depends on prompt recognisation and rescue pericardiocentesis.

Objective The aim of this report was to validate fluoroscopic heart silhouette characteristics associated with cardiac tamponade as a diagnostic method, and evaluate the safety and effectiveness of fluoroscopy-guided pericardiocentesis during catheter ablation.

Methods All cases of acute cardiac tamponade that occurred in the cardiac catheterisation laboratory during radiofrequency catheter ablation from March 2004 to November of 2009 were reviewed retrospectively.

Results Of 1832 catheter ablation procedures performed during a 5 -year period, $10(0.55 \%)$ were complicated by cardiac tamponade. Fluoroscopic examination confirmed the diagnosis in all 10 patients and demonstrated effusions before hypotension In 4 patients. All patients were stabilised by fluoroscopy-guided pericardiocentesis with placement of an indwelling catheter and autologous transfusion. The time interval between recognition of cardiac tamponade and completion of pericardiocentesis was $6.0 \pm 1.8 \mathrm{~min}$ (range 3-9 min). The mean aspirated blood volume was $437 \mathrm{ml}$ (range $110-1400 \mathrm{ml}$ ), and the mean autotransfused blood volume was $425 \mathrm{ml}$ (range 100-1384 ml). Surgical repair of the cardiac perforation was needed in one patient. No procedure-related death occurred. The ablation procedures were resumed and succeeded in 3 patients after pericardiocentesis.

Conclusion A reduction in the excursion of cardiac silhouette on fluoroscopy is an early diagnostic sign of cardiac tamponade during radiofrequency ablation. Fluoroscopy-guided pericardiocentesis is a safe and effective management strategy for cardiac tamponade developed in the cardiac catheterisation laboratory.

\section{e0568 FOLLOWUP OF FIVE PATIENTS WITH BRUGADA SYNDROME TREATED WITH ICD}

doi:10.1136/hrt.2010.208967.568

Yuan Binbin, Hasimu Buaijiaer, Zhang Kai. Department of Cardiology, Nanjing Benq Hospital, The Affiliated Hospital of Nanjing Medical University

Objective To investigate clinical symptoms, episodes of arrhythmias and its therapy in patients with Brugada syndrome treated with implantable cardioverter-defibrillator (ICD).

Methods Five patients with concealed Brugada syndrome (all male, mean age $41.6 \pm 10.14$ years) were treated with single-chamber ICD and followed up every three months. The time of onset, type of arrhythmia, treatments and its results of the episodes were investigated according to the data logs of the ICD.

Results The diagnosis of Brugada syndrome was made according to sodium channel blocker provocation test in four patients ( 2 by ajmaline, 2 by propafenone), and screen of new praecordial leads system in another case. Episodes of syncope in all patients and ventricular fibrillation in four cases were documented before ICD therapy. During electrophysiological study, ventricular fibrillation could be induced in three patients. During a follow-up of $22 \pm 18$ months, 75 episodes of ventricular fibrillation were documented. Among them 61 were terminated by 86 shocks successfully, 14 stopped spontaneously. One patient still experienced 4 episodes of syncope because of his increased defibrillation threshold. One patient had 26 times inappropriate shocks due to atrial fibrillation, which disappeared after we adjusted the protocol of the ICD. Another one had two episodes of syncope though no event was recorded in his ICD. Because the tilt test reached positive result, the diagnosis of vasovagal syncope was made.

Conclusion ICD implantation is a necessary and effective therapy for high risk patients with Brugada syndrome, and should be followed up regularly and programmed appropriately because of increased defibrillation threshold or inappropriate shocks.

\section{e0569 POSTCONDITIONING EFFECT ON REPERFUSION ARRHYTHMIA OF ST-SEGMENT ELEVATION ACUTE MYOCARDIAL INFARCTION}

doi:10.1136/hrt.2010.208967.569

Tongku Liu, Fuxiang Ding, Ming Gu. Department of Cardiology, Affiliated Hospital of Beihua University, Jilin, Jilin, China

Objective To study postconditioning effects during the first minutes of reperfusion in STEMI patients undergoing emergency percutaneous coronary intervention (PCI) on the myocardial reperfusion Arrhythmia.

Methods Between Oct. 2006 and Jan. 2009 at affiliated hospital of Beihua University, 64 patients diagnosed with STEMI undergoing emergency percutaneous transluminal coronary angioplasty (PTCA) and stenting within $12 \mathrm{~h}$ from onset of symptoms to open the infarct-related coronary artery, were randomly divided in two groups: the control group $(n=34)$ which were treated by implantation stent after PTCA, and the experimental group $(n=30)$ which were treated by ischaemic postconditioning within first minutes of reflow by 3 episodes of 30 -seconds inflation and 30 -seconds deflation of the angioplasty balloon. All patients were first onset of STEMI, and did not have the inverse perfusion from collateral circulation. Two groups were observed and compared with reperfusion arrhythmias within $5 \mathrm{~min}$ after beginning put into practice reperfusion.

Results In the control group and experimental group the incidence of frequent premature ventricular contraction (PVC) was dividedly $52.9 \%$ and $26.7 \%(p<0.05)$, paroxysmal ventricular tachycardia was dividedly $58.8 \%$ and $23.3 \%(p<0.05)$, nonparoxysmal ventricular tachycardia was dividedly $41.2 \%$ and $16.7 \%(p<0.05)$, ventricular fibrillation was dividedly $5.9 \%$ and $0 \%$, sinus bradycardia was dividedly $26.5 \%$ and $6.7 \%(p<0.05)$, sinus arrest was dividedly $20.6 \%$ and $3.3 \%(p<0.05)$, and atrioventricular block was dividedly $14.7 \%$ and $0 \% \quad(p<0.05)$. In postconditioning group there was significant reduction in the incidence of reperfusion arrhythmias.

Conclusions postconditioning in emergency PCI for STEMI can significantly reduce the incidence of myocardium reperfusion arrhythmias.

\section{e0570 CAN HATCH SCORE PREDICT RECURRENCE OF ATRIAL FIBRILLATION AFTER CATHETER ABLATION?}

doi:10.1136/hrt.2010.208967.570

Tang Ribo, Dong Jianzeng, Liu Xingpeng, Long Deyong, Yu Ronghui, Ma Changsheng. Beijing An Zhen Hospital

Background HATCH score $(1 *$ hypertention $+1 *$ age $>75]+2 *$ [stroke or transient ischaemic attack] $+1^{*}$ [chronic obstructive pulmonary disease] $+2 *$ [heart failure]) is an established predictor of progression from paroxysmal to persistent atrial fibrillation (AF). Whether atrial remodelling indexed by $\mathrm{HATCH}$ score could be a predictor of recurrence after catheter ablation of $\mathrm{AF}$ needs to be explored.

Methods The data of 608 consecutive AF patients who underwent an index circumferential pulmonary veins ablation were retrospectively analysed. Of these patients, 313 (51.5\%) patients had $\mathrm{HATCH}=0,225(37.0 \%)$ patients had $\mathrm{HATCH}=1,70(11.5 \%)$ patients had $\mathrm{HATCH} \geq 2$

Results The patients with $\mathrm{HATCH} \geq 2$ had significantly the largest left atrium size, the largest left ventricular end systolic diameter, and the lowest ejection fraction among the three HATCH categories. There were significant differences of the proportion of diabetes mellitus, statins medication, and angiotensin-converting enzyme inhibitors/angiotensin receptor blockers medications among the 
three HATCH categories. After a mean follow-up of $474 \pm 330$ days, the recurrence rate were $36.4 \%, 38.7 \%, 34.3 \%$, from $\mathrm{HATCH}=0$ to HATCH $\geq 2$ categories $(p=0.707)$. Univariate analysis revealed that nonparoxysmal AF, left atrium size, body mass index were predictors of AF recurrence. Multivariate analysis revealed that nonparoxysmal AF ( $\mathrm{HR}=1.43,95 \%$ CI 1.03 to $1.99, \mathrm{p}=0.031$ ) was the only independent predictor of AF recurrence. $\mathrm{HATCH}$ and left atrium size were not independent predictors of AF recurrence.

Conclusion HATCH has no value in prediction of AF recurrence after catheter ablation.

\section{Q0571 EFFECT OF VAGAL NERVE ON THE MONOPHASIC ACTION POTENTIAL OF VENTRICULAR OUTFLOW TRACT}

doi:10.1136/hrt.2010.208967.571

Chang Dong, Bai Xiaoyan, Zhang Shulong, Gao Lianjun, Yang Yanzong. First Affiliated Hospital of Dalian Medical University

Objective Vagal nerve may be related with idiopathetic ventricular tachycardia (IVT). The present study was aimed to investigate the effect of vagal nerve on the monophasic action potential (MAP) of ventricular outflow tract.

Methods Eight adult mongrel dogs were involved. Bilateral vagosympathetic tunks were decentralised for stimulation. Metoprolol was given to block sympathic effects. Three MAP recording electrode were placed at the left ventricular outflow tract (LVOT), right ventricular outflow tract (RVOT) and right ventricular apex (RVA) respectively through right femoral artery and vein. MAP was recorded at the LVOT, RVOT, RVA with or without vagal stimulation (VS) respectively.

Results MAP duration (MAPD) under VS was significantly shorter than baseline $(p>0.05)$. With or without VS, the MAPD at RVA were significantly shorter than that at RVOT and LVOT $(p<0.05)$, while there was no difference of MAPD between RVOT and LVOT. With VS, the abbreviation of MAPD at outflow tract was greater significantly than that at RVA (APD90 12.1 \pm 3.9 at RVOT, 14.8 \pm 5.5 at LVOT vs $8.3 \pm 4.1$ at RVA, $p<0.05)$, while there was no difference of MAPD between LVOT and RVOT ( $p>0.05$ ).

Conclusions VS could reduce MAPD significantly. With VS, the abbreviation of MAPD at outflow tract was greater significantly than that at RVA. It suggested that outflow tract may be sensible to vagal modulation, which might be related to the occurrence of IVT.

\section{e0572 COMPARISON OF PULMONARY VEINS OSTIUM, ANTRUM AND LEFT ATRIAL VOLUME IN PATIENTS WITH AND WITHOUT PAROXYSMAL ATRIAL FIBRILLATION}

doi:10.1136/hrt.2010.208967.572

Liu Min, Yang Yanzong, Zhang Shulong, Gao Lianjun. First Affiliated Hospital of Dalian Medical University

Objective To the date, catheter ablation basing on pulmonary vein (PVs) isolation is effective treatment for paroxysmal atrial fibrillation (PAF). In some experimental electrophysiological center, catheter ablation has been the first line for PAF. The aim of this study is to compare the variation of ostia, the antrum volume of pulmonary veins and the left atrial volume in patients with and without PAF. Methods We consecutively enrolled 28 and 35 patients with and without PAF (PAF group and control group). All the patients were taken intravenous injection of contrast medium before evaluated by 16-slice CT (MSCT). The 3D reconstruction of PVs and left atrium was transformed into AW4.2 system, and calculated the variation of pulmonary veins by Cardiac IQ software. Diameters of PVs ostia were measured by virtual endoscopy. The antrum volume of PVs and the left atrium volume were calculated by volume rendering. Results 3 patients $(10.7 \%)$ with PAF had common ostia or trunk of $\mathrm{PVs}$ and right middle PVs, respectively. We compared the maximum and minimum diameter of PVs ostia in PAF group with that in control group as follows: the maximum diameters, left superior PVs $(22.69 \pm 3.56$ vs $18.69 \pm 2.15 \mathrm{~mm}, \mathrm{p}<0.01)$, left inferior PVs $(18.40 \pm 2.50$ vs $16.96 \pm 2.07 \mathrm{~mm}, \mathrm{p}<0.05)$, right superior PVs $(20.78 \pm 3.46$ vs $19.26 \pm 2.55 \mathrm{~mm}, \quad \mathrm{p}=0.08)$, right inferior PVs $(20.19 \pm 4.39$ vs $16.90 \pm 1.75 \mathrm{~mm}, \mathrm{p}<0.01)$; the minimum diameter, left superior PVs $(16.18 \pm 3.60$ vs $11.12 \pm 2.55 \mathrm{~mm}, \mathrm{p}<0.01)$, left inferior PVs $(11.1 \pm 3.20$ vs $10.40 \pm 2.10 \mathrm{~mm}, \mathrm{p}<0.01)$, right superior PVs $(16.18 \pm 2.57$ vs $13.61 \pm 2.35 \mathrm{~mm}, \mathrm{p}<0.01)$, right inferior PVs $(16.26 \pm 3.16$ vs $13.29 \pm 2.20 \mathrm{~mm}, \mathrm{p}<0.01)$. The atrium volume of the bilateral PVs in PAF group was significantly larger than that in the control group (left, $3.53 \pm 0.74$ vs $2.74 \pm 0.49 \mathrm{~cm}^{3}, \quad \mathrm{p}<0.01$; right, $4.57 \pm 1.59$ vs $\left.3.54 \pm 1.01 \mathrm{~cm}^{3}, \mathrm{p}<0.01\right)$. The left atrial volume in PAF group was also significantly larger than that in control group ( $99.83 \pm 15.68$ vs $\left.88.24 \pm 18.21 \mathrm{~cm}^{3}, \mathrm{p}<0.05\right)$. The atrium volume of bilateral PVs justified with left atrial volume had no significant difference between the two groups.

Conclusion The ostial diameter and atrium volume of PVs were increased significantly, leading to the change for anatomy of atrial sleeves and distribution of autonomic nerve, and caused reentry or focal automaticity around PVs, which eventually initiated PAF. This study suggests that patients with PAF may exist potential structural disease of atria.

\section{E0573 IMPACT OF AGEING ON THE LEFT ATRIUM THROMBUS IN PATIENTS WITH NON-VALVULAR ATRIAL FIBRILLATION}

doi:10.1136/hrt.2010.208967.573

Tang Ri-bo, Liu Chang, Ma Chang-sheng, Dong Jian-zeng, Liu Xing-peng, Long De-yong, Yu Rong-hui. Beijing An Zhen Hospital

Objective This study sought to investigate the impact of ageing on left atrium thrombus in patients with non-valvular atrial fibrillation (AF). Methods Four hundred and twenty-one consecutive patients with AF underwent transesophageal echocardiography prior to AF ablation were included in a prospectively established database. The elderly group was defined as age more than 65 years old.

Results In all the 421 patients, 108 (25.6\%) were in the elderly group and $313(74.4 \%)$ were in the young group, with the mean age $70.0 \pm 3.7,52.1 \pm 8.5$, respectively. There was no significant difference of left atrium thrombus between the elderly group and the young group ( $4.6 \%$ vs $6.7 \%, p=0.439)$. It was shown there is less value for age predicting left atrium thrombus, the area under the receptoroperating curve was 0.494 . The mean age $(56.7 \pm 9.9$ vs $56.7 \pm 10.9$, $\mathrm{p}=0.989)$ and the proportion of the elderly $(19.2 \%$ vs $26.1 \%$, $\mathrm{p}=0.439$ ) did not differ significantly between the patients with left atrium thrombus and those without. After adjustment for ejection fraction, heart failure, hypertension, diabetes mellitus, angiotensinconverting enzyme inhibitors/angiotensin receptor blockers medication in logistics multivariate analysis, left atrium diameter, non-paroxysmal AF, prior thromboembolic history were independent risk factors of left atrium thrombus. However, age was not an independent risk factor of left atrium thrombus.

Conclusions Ageing itself was not a risk factor of left atrium thrombus in patients with non-vavular AF.

\section{e0574 HIGH-NORMAL THYROID FUNCTION AND RISK OF RECURRENCE OF ATRIAL FIBRILLATION AFTER CATHETER ABLATION}

doi:10.1136/hrt.2010.208967.574

Tang Ribo, Liu Dongling, Dong Jianzeng, Liu Xingpeng, Long Deyong, Yu Ronghui, Ma Changsheng. Beijing An Zhen Hospital

Background It has been shown that serum free thyroxine (FT4) concentration is independently associated with atrial fibrillation (AF) even in euthyroid persons. This study aimed to testify the 